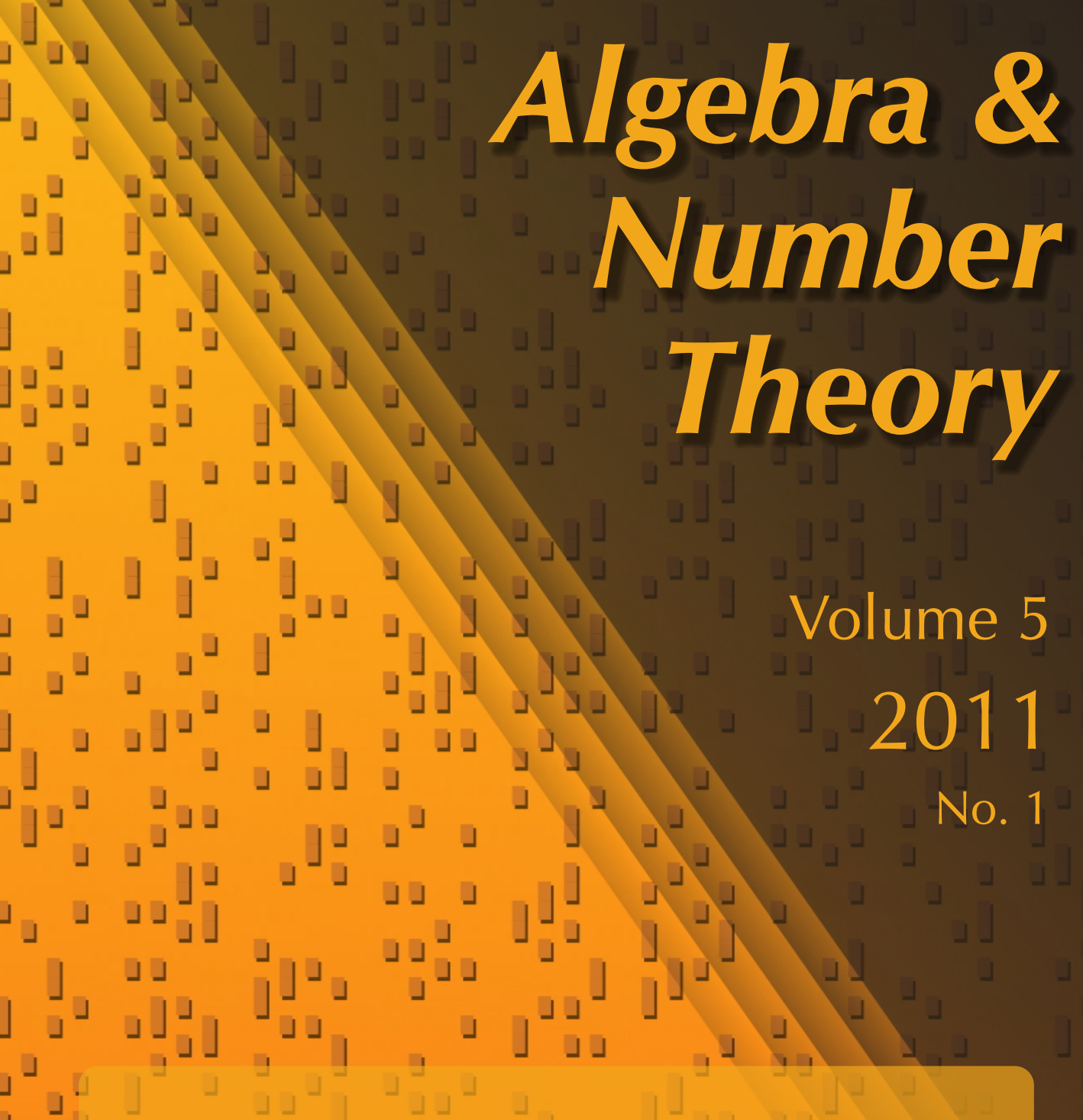

- Group algebra extensions of depth one

Robert Boltje and Burkhard Külshammer

\lrcorner

\lrcorner

\rfloor

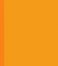

\lrcorner

Volume 5
2011
No.

.

」

لـ 


\title{
Group algebra extensions of depth one
}

\author{
Robert Boltje and Burkhard Külshammer
}

\begin{abstract}
A ring extension $A \subseteq B$ is said to have depth one if $B$ is isomorphic to a direct summand of $A^{n}$ as an $(A, A)$-bimodule, for some positive integer $n$. We prove group-theoretic characterizations of this property in the case $k H \subseteq k G$, where $H$ is a subgroup of a finite group $G$ and $k$ is a field. We determine when the source algebra of a block of $k G$ with defect group $P$ is a depth-one extension of $k P$.
\end{abstract}

\section{Introduction}

A ring extension is a unitary ring homomorphism $f: A \rightarrow B$ between two rings $A$ and $B$. In this situation, the ring $B$ can be viewed as an $(A, A)$-bimodule using the map $f$. A ring extension $f: A \rightarrow B$ is said to be of depth one (or centrally projective [Kadison 1999]) if $B$ is isomorphic, as an $(A, A)$-bimodule, to a direct summand of $A^{n}$ for some positive integer $n$. We write $B \mid A^{n}$ for this condition. Whenever $A$ is a unitary subring of $B$ and $f: A \rightarrow B$ is the inclusion map, we denote the corresponding ring extension by $A \subseteq B$.

To the best of our knowledge, centrally projective ring extensions were first considered by Hirata [1969]. The identification of centrally projective ring extensions with ring extensions of depth one appeared in [Kadison and Szlachányi 2003]. Ring extensions of higher depth were studied in [Kadison 2008], for example.

In this paper we try to answer the question of when a ring extension $k H \subseteq k G$ of group rings has depth one. Here and throughout this paper we denote by $k$ a commutative ring, by $G$ a group and by $H$ a subgroup of finite index in $G$. In [Boltje and Külshammer 2010] we considered the question of when the ring extension $k H \subseteq k G$ has depth two (that is, when $k G \otimes_{k H} k G \mid(k G)^{n}$ as $(k G, k H)$ bimodules, or equivalently as $(k H, k G)$-bimodules, for some positive integer $n)$. It turns out that this is equivalent to $H$ being normal in $G$, independently of $k$. If the ring extension $k H \subseteq k G$ has depth one it also has depth two, since one can apply the functor $k G \otimes_{k H^{-}}$to the relation $k G \mid(k H)^{n}$. In particular, $H$ has to be normal in $G$. The converse is not true in general, as our main results, Theorems 1.7 and 1.9,

MSC2000: primary 20C05; secondary 19A22, 16D90, 16D20, 20C20.

Keywords: depth-one ring extension, centrally projective ring extension, depth-two ring extension, symmetric Frobenius extension, $p$-hypoelementary group, nilpotent block, source algebra, trivial source module. 
show. In these theorems we have to assume that $k$ is a field (or a complete discrete valuation ring of characteristic 0 and positive residual characteristic $p$ ). In both cases the depth-one condition is equivalent to a purely group theoretic condition on the inclusion $H \leqslant G$, namely that $G=H C_{G}(X)$, for every cyclic subgroup $X$ of $H$ in the characteristic 0 case, and for every $p$-hypoelementary subgroup $X$ of $H$ in the modular case (see Remark 1.8 for a definition of $p$-hypoelementary groups). Therefore, the group theoretic depth-one condition does depend on the base ring $k$. We do not have a group theoretic reformulation in the case $k=\mathbb{Z}$.

At the end we study the depth-one condition for the ring extension $F P \rightarrow A$, where $P$ is the defect group of a block of a group algebra over a field $F$ of positive characteristic $p$, and $A$ is a source algebra of the block.

\section{Depth one for group algebra extensions}

1.1. Assume that $g$ belongs to $N_{G}(H)$, the normalizer of $H$ in $G$. Then

$$
c_{g^{-1}}: k H \rightarrow k H, \quad a \mapsto g^{-1} a g
$$

is a $k$-algebra automorphism of $k H$. Restriction along this automorphism defines a functor

$$
C_{g}:{ }_{k H} \operatorname{Mod} \rightarrow{ }_{k H} \operatorname{Mod}, \quad M \mapsto{ }^{g} M
$$

on the category of left $k H$-modules. More explicitly, for $M \in{ }_{k H}$ Mod, the left $k H$-module ${ }^{g} M$ is defined to be equal to $M$ as an abelian group, and it is endowed with the module structure $a * m:=\left(g^{-1} a g\right) \cdot m$, where "." denotes the original $k H$-module structure of $M$. The functor $C_{g}$ maps a homomorphism $f: M_{1} \rightarrow M_{2}$ in ${ }_{k H}$ Mod to ${ }^{g} f:=f:{ }^{g} M_{1} \rightarrow{ }^{g} M_{2}$.

If $C_{g}$ is naturally equivalent to the identity functor on ${ }_{k H}$ Mod, we say that $g$ acts trivially on ${ }_{k H}$ Mod. For this paper, we say that $G$ acts trivially on ${ }_{k H}$ Mod if $H$ is normal in $G$ and $g$ acts trivially on ${ }_{k H}$ Mod for every $g \in G$. Note that $H$ acts trivially on ${ }_{k H}$ Mod. This is also an immediate consequence of the next proposition.

The subset $k g H=g k H=k H g$ of $k G$ is a $(k H, k H)$-subbimodule of $k G$. It is isomorphic to ${ }^{(g, 1)} k H$ if we view $(k H, k H)$-bimodules $M$ as left $k[H \times H]$-modules via

$$
\left(h_{1}, h_{2}\right) \cdot m:=h_{1} m h_{2}^{-1} \quad \text { for } h_{1}, h_{2} \in H \text { and } m \in M .
$$

Assume that $R$ is an arbitrary ring and that $\alpha$ is an automorphism of $R$. Let $R_{\alpha}$ denote the $(R, R)$-bimodule that equals $R$ as abelian group but has the twisted action $a x b:=\alpha(a) x b$, for $a, x, b \in R$. It is well-known and straightforward to prove that $\alpha$ is an inner automorphism if and only if $R_{\alpha}$ is isomorphic to $R$ as an $(R, R)$-bimodule. It is also equivalent to $\alpha$ acting trivially on ${ }_{R}$ Mod. The following proposition is a special case, and we leave its proof to the reader. 
Proposition 1.2. For $g \in N_{G}(H)$, the following are equivalent:

(i) $k g H \cong k H$ as $(k H, k H)$-bimodules.

(ii) There exists a unit $u$ of $k H$ such that $\mathrm{gag}^{-1}=u a u^{-1}$ for all $a \in k H$.

(iii) $g$ acts trivially on ${ }_{k H}$ Mod.

For every subset $X$ of $H$ we denote by $C_{G}(X)$ the centralizer of $X$ in $G$.

Corollary 1.3. If $G=H C_{G}(H)$, then the ring extension $k H \subseteq k G$ has depth one. Conversely, if the ring extension $k H \subseteq k G$ has depth one, then $H$ is normal in $G$.

Proof. Suppose first that $G=H C_{G}(H)$. Then every $g \in G$ satisfies condition (ii). Using condition (i) together with the decomposition $k G=\bigoplus_{g H \in G / H} \mathrm{kgH}$ into $(k H, k H)$-subbimodules, the first assertion follows. The second assertion was already observed in the introduction.

1.4. If $\Lambda$ is a $k$-order (that is, a $k$-algebra that is finitely generated and projective as a $k$-module) we say that the Krull-Schmidt theorem holds for $\Lambda$-lattices if the following two properties hold for every $\Lambda$-module $M$ that is finitely generated and projective as a $k$-module:

- $M$ has a decomposition $M=U_{1} \oplus \cdots \oplus U_{r}$ into indecomposable $\Lambda$-submodules, and

- if $M=U_{1} \oplus \cdots \oplus U_{r}=V_{1} \oplus \cdots \oplus V_{s}$ are two decompositions into indecomposable $\Lambda$-submodules, then $r=s$ and there exists a permutation $\sigma$ of $\{1, \ldots, r\}$ such that $U_{i} \cong V_{\sigma(i)}$ for all $i \in\{1, \ldots, r\}$.

If $k$ is a field or a complete discrete valuation ring, then the Krull-Schmidt theorem holds for every $k$-order $\Lambda$ [Curtis and Reiner 1981, Theorem 6.12].

Proposition 1.5. Assume that $G$ is finite and that the Krull-Schmidt theorem holds for $k[H \times H]$-lattices. The following are equivalent:

(i) $k H \subseteq k G$ is a ring extension of depth one.

(ii) $H$ is normal in $G$ and $k g H \cong k H$ as $(k H, k H)$-bimodules for every $g \in G$.

(iii) $H$ is normal in $G$ and $k G \cong(k H)^{[G: H]}$ as $(k H, k H)$-bimodules.

(iv) $k G \cong(k H)^{[G: H]}$ as $(k H, k H)$-bimodules.

Proof. (i) $\Rightarrow$ (ii): Since the ring extension $k H \subseteq k G$ has depth one, Corollary 1.3 implies that $H$ is normal in $G$. Therefore, for every $g \in G$, one has $k g H|k G|(k H)^{n}$ as $(k H, k H)$-bimodules for some positive integer $n$. Since the Krull-Schmidt theorem holds for $k[H \times H]$-lattices, every indecomposable direct summand of the $k[H \times H]$-module $\mathrm{kgH}$ is isomorphic to an indecomposable direct summand of the $k[H \times H]$-module $k H$. But the indecomposable direct summands of $k H$ are the blocks of $k H$, and they are pairwise nonisomorphic. Since $\mathrm{kgH}$ is isomorphic to 
${ }^{(g, 1)} k H$ and since $C_{(g, 1)}:{ }_{k[H \times H]} \operatorname{Mod} \rightarrow{ }_{k[H \times H]}$ Mod is a category equivalence, the indecomposable direct summands of $\mathrm{kgH}$ are also pairwise nonisomorphic. Thus, we can decompose $\mathrm{kgH}$ and $\mathrm{kH}$ multiplicity-free into a direct sum of indecomposable $k[H \times H]$-submodules. The number of these summands coincides, since $\mathrm{kgH} \cong{ }^{(g, 1)} \mathrm{kH}$. Since every summand of $\mathrm{kgH}$ occurs as a summand of $k H$, we can conclude that $k g H \cong k H$ as $(k H, k H)$-bimodules.

(ii) $\Rightarrow$ (iii): This follows from the decomposition $k G=\bigoplus_{g H \in G / H} \mathrm{kgH}$ into $(k H, k H)$-subbimodules.

(iii) $\Rightarrow$ (iv): This is trivial.

(iv) $\Rightarrow$ (i): This is immediate from the definition of depth one.

Remark 1.6. In the proof of Proposition 1.5, the Krull-Schmidt property is only used for permutation $k[H \times H]$-modules. By [Boltje and Glesser 2007, Theorem 1.6], the four conditions in Proposition 1.5 are still equivalent when $k$ is a local domain containing a root of unity of order $e$, where $e$ is defined as the exponent $\exp (H)$ of $H$ in the case that $k$ has characteristic 0 , and as the $p^{\prime}$-part of $\exp (H)$ in the case that $k$ has positive characteristic $p$.

Next we will study the case where $k$ is a field of characteristic 0 and $H$ is a finite group. In this case we will denote by $\bar{k}$ an algebraic closure of $k$ and by $\operatorname{Irr}(H)$ the set of irreducible characters of $\bar{k} H$. Recall that $N_{G}(H)$ acts from the left on $\operatorname{Irr}(H)$ via $\chi \mapsto{ }^{g} \chi$ for $g \in N_{G}(H)$, where ${ }^{g} \chi(h)=\chi\left(g^{-1} h g\right)$, for $h \in H$. Recall also that $N_{G}(H)$ acts on the set of conjugacy classes of $H$ via $\mathscr{K} \mapsto g \%$, where $g \mathscr{K}=\left\{g h g^{-1} \mid h \in \mathscr{K}\right\}$ for a conjugacy class $\mathscr{K}$ of $H$.

Theorem 1.7. Assume that $k$ is a field of characteristic 0 and that $G$ is finite. The following are equivalent:

(i) $k H \subseteq k G$ is a ring extension of depth one.

(ii) $H$ is normal in $G$ and $G$ acts trivially on $\operatorname{Irr}(H)$.

(iii) $H$ is normal in $G$ and $G$ acts trivially on the set of conjugacy classes of $H$.

(iv) For every cyclic subgroup $X$ of $H$ one has $G=H C_{G}(X)$.

Proof. (i) $\Longleftrightarrow$ (ii): Using Corollary 1.3, we may assume that $H$ is normal in $G$. Using Propositions 1.2 and 1.5, one sees that it suffices to show that, for every $g \in G$, the $(k H, k H)$-bimodules $k g H$ and $k H$ are isomorphic if and only if $g$ acts trivially on $\operatorname{Irr}(H)$. By the Deuring-Noether theorem [Nagao and Tsushima 1989, Theorem II.3.1], $k g H \cong k H$ as $(k H, k H)$-bimodules if and only if $\bar{k} g H \cong \bar{k} H$ as $(\bar{k} H, \bar{k} H)$-bimodules. By Proposition 1.2, the latter is equivalent to $g$ acting trivially on ${ }_{\bar{k}}{ }$ Mod, which implies that $g$ acts trivially on $\operatorname{Irr}(H)$. Conversely, if ${ }^{g} \chi=\chi$ for every $\chi \in \operatorname{Irr}(H)$ then $\bar{k} g H \cong \bar{k} H$ as $\bar{k}[H \times H]$-modules, since the 
character of $\bar{k} H$ is equal to $\sum_{\chi \in \operatorname{Irr}(H)} \chi \times \bar{\chi}$ and the character of $\bar{k} g H$ is equal to

$$
{ }^{(g, 1)}\left(\sum_{\chi \in \operatorname{Irr}(H)} \chi \times \bar{\chi}\right)=\sum_{\chi \in \operatorname{Irr}(H)} g_{\chi} \times \bar{\chi}=\sum_{\chi \in \operatorname{Irr}(H)} \chi \times \bar{\chi} .
$$

(ii) $\Longleftrightarrow$ (iii): This follows immediately from Brauer's permutation lemma [Nagao and Tsushima 1989, Lemma III.2.19].

(iii) $\Rightarrow$ (iv): Let $X=\langle x\rangle$ be a cyclic subgroup of $H$ and let $g$ be an element of $G$. Since $g x g^{-1}$ lies in the same conjugacy class as $x$, there exists $h \in H$ such that $g x g^{-1}=h x h^{-1}$. This implies $h^{-1} g \in C_{G}(x)$ and $g \in h C_{G}(x) \subseteq H C_{G}(X)$.

(iv) $\Rightarrow$ (ii): Condition (iv) implies immediately that $H$ is normal in $G$. Now let $g \in G, \chi \in \operatorname{Irr}(H)$ and $x \in H$. Then there exists $h \in H$ and $c \in C_{G}(x)$ such that $g=h c$. Hence $\chi\left(g x g^{-1}\right)=\chi\left(h c x c^{-1} h^{-1}\right)=\chi\left(h x h^{-1}\right)=\chi(x)$. Thus ${ }^{-1} \chi=\chi$ and $\chi={ }^{g} \chi$.

Remark 1.8. Next we study the depth-one condition for the ring extension $k H \subseteq$ $k G$ in the case where $k$ is a field of positive characteristic $p$, or where $k$ is a complete discrete valuation ring of characteristic 0 and positive residual characteristic p. We will need the theory of species developed by Benson and Parker [1998, Section 5.5]. For this remark assume that $G$ is finite and that $k$ contains a root of unity whose order is equal to $\exp (G)$ if $k$ has characteristic 0 , and to the $p^{\prime}$-part of $\exp (G)$ if $k$ has characteristic $p$. Let $S$ and $T$ be finite left $G$-sets. We denote the corresponding permutation $k G$-modules by $k S$ and $k T$. The goal of this remark is to derive a criterion for $k S$ being isomorphic to $k T$. Denote by $\mathscr{H}_{p}(G)$ the set of $p$-hypoelementary subgroups $E$ of $G$, that is, subgroups $E$ that have a normal (Sylow) $p$-subgroup $P$ such that $E / P$ is cyclic. We claim that

$$
k S \cong k T \text { as } k G \text {-modules } \Longleftrightarrow\left|S^{E}\right|=\left|T^{E}\right| \text { for all } E \in \mathscr{H}_{p}(G),
$$

where $\left|S^{E}\right|$ denotes the cardinality of the set $S^{E}$ of $E$-fixed points on $S$. In order to see this equivalence, it suffices to show that

$$
s_{E, g}(k S)=\left|S^{E}\right|
$$

for all $E \in \mathscr{H}_{p}(G)$ and all $p^{\prime}$-elements $g \in E$ such that $g P$ generates $E / P$, where $P$ denotes the Sylow $p$-subgroup of $E$. For a definition of $s_{E, g}$ see [Benson 1998, Section 5.5]. Since $s_{E, g}(k S)=s_{E, g}\left(\operatorname{Res}_{E}^{G}(k S)\right)$, we may assume that $G=E$. Moreover, since $s_{E, g}$ is additive, we may assume that $S$ is a transitive $E$-set, that is, $S=E / D$ for some subgroup $D$ of $E$. Then $k S \cong \operatorname{Ind}_{D}^{E}(k)$. If $P$ is not contained in $D$, then no indecomposable direct summand of $k S$ has vertex $P$, and both sides of Equation (1.8.b) are equal to 0 . If $P \leqslant D<E$, the Brauer species of $\operatorname{Ind}_{D}^{E}(k)$ at $g$ equals 0 , since $g \notin D$, and again both sides in Equation (1.8.b) are equal to 0 . Finally, if $D=E$, it is immediate that both sides of the equation are equal to 1 . 
In the next theorem we will apply the criterion in (1.8.a) to the $H \times H$-sets $g H$ and $H$ for $g \in N_{G}(H)$.

For a subgroup $X$ of $H$, we set $\Delta X:=\{(x, x) \mid x \in X\} \leqslant H \times H$.

Theorem 1.9. Assume that $G$ is finite and that $k$ is a field of characteristic $p>0$ or a complete discrete valuation ring of characteristic 0 with residual characteristic $p>0$. The following are equivalent:

(i) The ring extension $k H \subseteq k G$ has depth one.

(ii) $H$ is normal in $G$, and $\left|(g H)^{E}\right|=\left|H^{E}\right|$ for all $g \in G$ and $E \in \mathscr{H}_{p}(H \times H)$.

(iii) $H$ is normal in $G$, and $\left|(g H)^{\Delta X}\right|=\left|H^{\Delta X}\right|$ for all $g \in G$ and $X \in \mathscr{H}_{p}(H)$.

(iv) For all $X \in \mathscr{H}_{p}(H)$ one has $G=C_{G}(X) H$.

Proof. (i) $\Longleftrightarrow$ (ii): By Corollary 1.3, we may assume that $H$ is normal in $G$. Now the equivalence of (i) and (ii) follows immediately from Proposition 1.5 and Remark 1.8 applied to the $k[H \times H]$-modules $k g H$ and $k H$, for $g \in G$. In fact, by [Benson 1998, Corollary 3.11.4(i)] and the Deuring-Noether theorem [Nagao and Tsushima 1989, Theorem II.3.1], we have $k g H \cong k H$ as $k[H \times H]$-modules if and only if $k^{\prime} g H \cong k^{\prime} H$ as $k^{\prime}[H \times H]$-modules, where $k^{\prime}$ is obtained from $k$ by adjoining a root of unity whose order is equal to $\exp (H)$ if $k$ has characteristic 0 , and to the $p^{\prime}$-part of $\exp (H)$ if $k$ has characteristic $p$.

(ii) $\Rightarrow$ (iii): This is trivial.

(iii) $\Rightarrow$ (iv): Let $g \in G$ and let $X \in \mathscr{H}_{p}(H)$. Since $1 \in H^{\Delta X}$, the set $(g H)^{\Delta X}$ is nonempty. Let $h \in H$ such that $g h \in(g H)^{\Delta X}$. Then $g h \in C_{G}(X)$ and $g \in C_{G}(X) H$.

(iv) $\Rightarrow$ (ii): From (iv) we have immediately that $H$ is normal in $G$. Now let $g \in G$ and let $E \in \mathscr{H}_{p}(H)$. The $H \times H$-sets $H$ and $g H$ are transitive. The stabilizer of $1 \in H$ is $\Delta H$ and the stabilizer of $g \in g H$ is ${ }^{(g, 1)} \Delta H$. One has $\left|H^{E}\right|=0=\left|(g H)^{E}\right|$ unless $E$ is $H \times H$-conjugate to a subgroup of $\Delta H$ or ${ }^{(g, 1)} \Delta H$. Since the number of fixed points does not change if we replace $E$ by an $H \times H$-conjugate of $E$, we may assume that $E \leqslant \Delta H$ or $E \leqslant{ }^{(g, 1)} \Delta H$. We first assume that $E \leqslant \Delta H$. Then $E=\Delta X$ for some $X \in \mathscr{H}_{p}(H)$. Since $g \in C_{G}(X) H$, we can write $g=c h$ with $c \in C_{G}(X)$ and $h \in H$. Then $g H=c H$, and for $h^{\prime} \in H$ we have

$$
h^{\prime} \in H^{\Delta X} \Longleftrightarrow h^{\prime} \in C_{G}(X) \Longleftrightarrow c h^{\prime} \in C_{G}(X) \Longleftrightarrow c h^{\prime} \in(c H)^{\Delta X} .
$$

It follows that $\left|H^{\Delta X}\right|=\left|(c H)^{\Delta X}\right|=\left|(g H)^{\Delta X}\right|$. Finally, if $E \leqslant{ }^{(g, 1)} \Delta H$, then ${ }^{\left({ }^{-1}, 1\right)} E=\Delta X$ for some $X \in \mathscr{H}_{p}(H)$. Again we can write $g=c h$ with $c \in C_{G}(X)$ and $h \in H$. Then $g=h^{\prime} c$ with $h^{\prime}=g h g^{-1} \in H$ and $E={ }^{(g, 1)}(\Delta X)={ }^{\left(h^{\prime} c, 1\right)}(\Delta X)=$ ${ }^{\left(h^{\prime}, 1\right)}(\Delta X)$ is $H \times H$-conjugate to $\Delta X$. By the first case, this implies

$$
\left|(g H)^{E}\right|=\left|(g H)^{\Delta X}\right|=\left|H^{\Delta X}\right|=\left|H^{E}\right| .
$$


Remark 1.10. In the case $k=\mathbb{Z}$, we do not know if there is a similar equivalence (i) $\Longleftrightarrow$ (iv) as in Theorem 1.9 with $\mathscr{H}_{p}(H)$ replaced by some other set $\mathscr{T}(H)$ of subgroups of $H$. Even if there existed such a set $\mathscr{S}(H)$, we don't have a good guess what it should be.

If $\mathbb{Z} H \subseteq \mathbb{Z} G$ has depth one, then $k H \subseteq k G$ has depth one for every commutative ring $k$ (by scalar extension). In particular, this implies that $G=H C_{G}(X)$ for every $p$-hypoelementary subgroup $X$ of $H$ for all primes $p$. We do not know if the converse holds. On the other hand, if $G=H C_{G}(H)$, then $\mathbb{Z} H \subseteq \mathbb{Z} G$ has depth one by Corollary 1.3. However, the converse is not true. In fact, by [Hertweck 2001, Theorem A], there exist a finite group $H$ (metabelian of order $2^{25} \cdot 97^{2}$ ), a noninner automorphism $g$ of $H$, and a unit $u$ of $\mathbb{Z} H$ with $g(a)=u a u^{-1}$. We set $G:=H \rtimes\langle g\rangle$. By Proposition 1.2 , we obtain $\mathbb{Z} g^{i} H \cong \mathbb{Z} H$ as $(\mathbb{Z} H, \mathbb{Z} H)$-bimodules for every integer $i$. This implies that $\mathbb{Z} G=\bigoplus_{x H \in G / H} \mathbb{Z} x H \cong(\mathbb{Z} H)^{[G: H]}$ and that $\mathbb{Z} H \subseteq \mathbb{Z} G$ has depth one. But $g \notin C_{G}(H) H$, since $g$ is not an inner automorphism of $H$. This shows that if, for each finite group $H$, there exists a set of subgroups $\mathscr{S}(H)$ of $H$ that replaces $\mathscr{H}_{p}(H)$ in Theorem 1.9(iv) in the case $k=\mathbb{Z}$, then $H \notin \mathscr{Y}(H)$ for Hertweck's group $H$.

\section{Depth one for source algebras of blocks}

2.1. Let $G$ be a finite group, let $p$ be a prime, and let $(K, R, F)$ be a $p$-modular system. Thus, $R$ is a complete discrete valuation ring of characteristic zero, $K$ is the field of fractions of $R$, and $F$, the residue field of $R$, has characteristic $p$. We assume that $R$ contains a root of unity of order $\exp (G)$ and that $F$ is algebraically closed. Then $K$ and $F$ are splitting fields for $K G$ and $F G$, respectively. For an $R$-order $A$, we denote by $\bar{A}$ the finite-dimensional $F$-algebra $F \otimes_{R} A$. In the following, let $k \in\{R, F\}$.

In this section, we will consider the depth-one condition for blocks and source algebras. For general background, we refer to the books [Thévenaz 1995] and [Külshammer 1991]. For the convenience of the reader, we recall some of the basic concepts.

An interior $G$-algebra over $k$ consists of a $k$-order $A$ and a group homomorphism $i: G \rightarrow A^{\times}$, where $A^{\times}$denotes the group of units of $A$. In this case, we will consider the $k$-linear extension $k G \rightarrow A$ of $i$ as a ring extension. Two interior $G$-algebras $A_{1}$ and $A_{2}$ are called isomorphic if there exists an isomorphism $f: A_{1} \rightarrow A_{2}$ commuting with the structural maps $i_{1}: G \rightarrow A_{1}^{\times}$and $i_{2}: G \rightarrow A_{2}^{\times}$.

If $A$ is an interior $G$-algebra, then a point of a subgroup $H$ of $G$ on $A$ is an $\left(A^{H}\right)^{\times}$-conjugacy class $\beta$ of primitive idempotents in the subalgebra

$$
A^{H}:=\{a \in A \mid h a=a h \text { for all } h \in H\}
$$

of $A$. In this case the pair $(H, \beta)=: H_{\beta}$ is called a pointed group on $A$. 
The point $\beta$ of $H$ on $A$ is called local if $\beta \nsubseteq \operatorname{Tr}_{L}^{H}\left(A^{L}\right)$ for every proper subgroup $L$ of $H$; here $\operatorname{Tr}_{L}^{H}: A^{L} \rightarrow A^{H}, a \mapsto \sum_{h L \in H / L} h a h^{-1}$ is the relative trace map. If $\beta$ is a local point of $H$ on $A$ then $H_{\beta}$ is called a local pointed group on $A$. One can show that in this case $H$ has to be a $p$-group.

Let $H_{\beta}$ and $L_{\gamma}$ be pointed groups on $A$. We write $L_{\gamma} \leq H_{\beta}$ if $L \leqslant H$ and $j A j \subseteq i A i$ for suitable idempotents $i \in \beta, j \in \gamma$. This defines a partial order on the set of pointed groups on $A$. The group $G$ acts by conjugation on the set of all pointed groups $H_{\beta}$ on $A$, and this action is compatible with the partial order relation. We denote by $N_{G}\left(H_{\beta}\right)$ the stabilizer of $H_{\beta}$ in $G$. Thus, $N_{G}\left(H_{\beta}\right)$ is a subgroup of $N_{G}(H)$.

A block of $k G$ is an indecomposable direct summand $B$ of $k G$, considered as a ( $k G, k G$ )-bimodule. In this case $B$ is a $k$-order in its own right. We consider $B$ as an interior $G$-algebra via the group homomorphism $G \rightarrow B^{\times}, g \mapsto g 1_{B}=1_{B} g$. Then $\alpha:=\left\{1_{B}\right\}$ is a point of $G$ on $B$ and we consider $G_{\alpha}$ as a pointed group on $B$.

The maximal local pointed groups $P_{\gamma} \leqslant G_{\alpha}$ are called defect pointed groups of $G_{\alpha}$ (and of $B$ ). They are unique up to conjugation in $G$. If $P_{\gamma}$ is a defect pointed group on $B$, then $P$ is also called a defect group of $B$. For $i \in \gamma$, the $k$-order $B_{\gamma}=i B i=i k G i$ is called a source algebra of $B$. One can show that $B i B=B$, so that $B$ and $i B i$ are Morita equivalent $k$-orders via multiplication with $i$. The source algebra $i B i$ will always be considered as an interior $P$-algebra via the map $P \rightarrow(i B i)^{\times}, x \mapsto i x=x i$.

The block $B$ is called nilpotent if $N_{G}\left(Q_{\delta}\right) / C_{G}(Q)$ is a $p$-group for every local pointed group $Q_{\delta} \leq G_{\alpha}$ on $B$. (Note that indeed $C_{G}(Q) \subseteq N_{G}\left(Q_{\delta}\right)$ here.) Puig [1988] determined the structure of the source algebra of a nilpotent block. It is a consequence of his results that every nilpotent block has a unique simple module in characteristic $p$, up to isomorphism. We will make use of Puig's results in the following theorem.

Theorem 2.2. Let $B$ be a block of $R G$ with defect pointed group $P_{\gamma}$, and let $B_{\gamma}$ be a corresponding source algebra. Then the following assertions are equivalent:

(i) The ring extension $F P \rightarrow \overline{B_{\gamma}}$ defined by the canonical map $P \rightarrow \overline{B_{\gamma}} \times$ has depth one.

(ii) $B_{\gamma}$ and $R P$ are isomorphic as interior P-algebras.

(iii) $B$ is a nilpotent block, and the unique simple $\bar{B}$-module $M$ has a trivial source. Proof. (i) $\Rightarrow$ (ii): Suppose that the ring extension $F P \rightarrow \overline{B_{\gamma}}$ has depth one. Then $\overline{B_{\gamma}} \mid(F P)^{n}$ as an $(F P, F P)$-bimodule, for some positive integer $n$. Thus every indecomposable direct summand of the $(F P, F P)$-bimodule $\overline{B_{\gamma}}$ is isomorphic to $F P$. Hence [Thévenaz 1995, Theorem 44.3] implies that $N_{G}\left(P_{\gamma}\right)=P C_{G}(P)$ and $\overline{B_{\gamma}} \cong F P$, as an $(F P, F P)$-bimodule; in particular, we have $\operatorname{rk}_{R}\left(B_{\gamma}\right)=\operatorname{dim}_{F} \overline{B_{\gamma}}=$ $|P|$. The same theorem now implies that $B_{\gamma} \cong R P$ as interior $P$-algebras. 
(ii) $\Rightarrow$ (iii): Suppose that $B_{\gamma}$ and $R P$ are isomorphic interior $P$-algebras. Then a result by Puig [1988, Theorem 1.6] implies that the block $B$ is nilpotent [Thévenaz 1995, Remark 50.10]. We write $\overline{B_{\gamma}}=i F G i$, where $i$ is a primitive idempotent in $(F G)^{P}$. Since every block has at least one simple module whose vertices are defect groups of the block, $P$ is a vertex of the unique simple $\bar{B}$-module $M$. By [Thévenaz 1995, Proposition 38.3], $M$ has an $F P$-source $V$ such that $V \mid i M$, as an $F P$-module. Since $\bar{B}$ and $\overline{B_{\gamma}}$ are Morita equivalent via multiplication with $i$, the $\overline{B_{\gamma}}$-module $i M$ is simple. Since $\overline{B_{\gamma}} \cong F P, i M$ is trivial as an $F P$-module, and so is $V$.

(iii) $\Rightarrow$ (i): Suppose that $B$ is nilpotent and that the unique simple $\bar{B}$-module $M$ has a trivial source. Then $M$ has vertex $P$, as above, and a result by Puig [Thévenaz 1995, Theorem 50.6] implies that $\overline{B_{\gamma}} \cong S \otimes_{F} F P$ as interior $P$-algebras, where $S$ is an interior $P$-algebra that is simple as an $F$-algebra. (The tensor product of two interior $P$-algebras is again an interior $P$-algebra via the diagonal map.) As above, we write $\overline{B_{\gamma}}=i F G i$, where $i$ is a primitive idempotent in $(F G)^{P}$. Since $\bar{B}$ and $\overline{B_{\gamma}}$ are Morita equivalent via multiplication with $i$, the module $i M$ is the unique simple $\overline{B_{\gamma}}$-module, up to isomorphism. Thus, $S$ and $\operatorname{End}_{F}(i M)$ are isomorphic interior $P$-algebras; in particular, $S^{P} \cong \operatorname{End}_{F P}(i M)$ as $F$-algebras. But $S^{P}$ is a local ring (since $\bar{B}_{\gamma}{ }^{P}$ is), so $i M$ is indecomposable as an $F P$-module. On the other hand, [Thévenaz 1995, Proposition 38.3] implies that $i M$ has a direct summand, as an $F P$-module, which is a source of $M$. Thus $\operatorname{dim}_{F} i M=1$. Hence $\operatorname{dim}_{F} S=1$, so $S \cong F$ and $\overline{B_{\gamma}} \cong F P$. In particular, the ring extension $F P \rightarrow \overline{B_{\gamma}}$ has depth one.

2.3. It would be interesting to have a similar description of the depth-two condition for source algebras of blocks. The goal of this subsection is to show that $R P \rightarrow B_{\gamma}$ (and also $F P \rightarrow \overline{B_{\gamma}}$ ) is a symmetric Frobenius extension, so that the left and right depth-two conditions are equivalent [Kadison and Szlachányi 2003, Proposition 6.4].

Recall from [Kadison 1999, Theorem I.1.2] that a ring extension $f: \Gamma \rightarrow \Delta$ is called a Frobenius extension if there exist a $(\Gamma, \Gamma)$-bimodule homomorphism $E: \Delta \rightarrow \Gamma$ and elements $x_{j}, y_{j} \in \Delta, j=1, \ldots, n$, such that

$$
\sum_{j=1}^{n} x_{j} E\left(y_{j} a\right)=a=\sum_{j=1}^{n} E\left(a x_{j}\right) y_{j}
$$

for all $a \in \Delta$. If in addition

$$
E(c a)=E(a c)
$$

holds for all $a \in \Delta$ and $c \in C_{\Delta}(\Gamma)$, then one calls the extension $f: \Gamma \rightarrow \Delta$ a symmetric Frobenius extension.

If $\Gamma \subseteq \Delta$ is a symmetric Frobenius extension and $e$ is an idempotent in $C_{\Delta}(\Gamma)$, then $e \Gamma e \subseteq e \Delta e$ is a symmetric Frobenius extension. In fact, if $E: \Delta \rightarrow \Gamma$ satisfies 
(2.3.a) and (2.3.b), then it is easy to verify that $\widetilde{E}: e \Delta e \rightarrow e \Gamma e, a \mapsto e E(a) e$ satisfies

$$
\sum_{j=1}^{n} e x_{j} e \widetilde{E}\left(e y_{j} e a\right)=a=\sum_{j=1}^{n} \widetilde{E}\left(a e x_{j} e\right) e y_{j} e
$$

for all $a \in e \Delta e$. Moreover, Equation (2.3.b) implies $\widetilde{E}(c a)=\widetilde{E}(a c)$ for all $a \in e \Delta e$ and $c \in C_{e \Delta e}(e \Gamma e)=e C_{\Delta}(\Gamma) e$.

If $H$ is a subgroup of $G$, then $k H \subseteq k G$ is a symmetric Frobenius extension. In fact, one can choose for $E: k G \rightarrow k H$ the canonical projection, and for $x_{j}$ and $y_{j}$, coset representatives of $G / H$ and their inverses. Thus, if $e$ is an idempotent in $(k G)^{H}$, then also $e k H e \rightarrow e k G e$ is a symmetric Frobenius extension. This holds even over arbitrary commutative rings $k$.

Now our claim follows by specializing to $H=P$ and $e=1_{B_{\gamma}}\left(\right.$ or $e=1_{\overline{B_{\gamma}}}$ ), and noting that $k P \rightarrow e k P e, a \mapsto e a e=e a=a e$ is an isomorphism of $k$-algebras.

By the preceding discussion, we do not need to distinguish between the left and the right depth-two condition in the following proposition.

Proposition 2.4. Let $B$ be the principal block of $R G$, and let $P_{\gamma}$ be a maximal local pointed group on $B$ (so that $P$ is a Sylow p-subgroup of $G$ ). Set $E:=$ $N_{G}\left(P_{\gamma}\right) / P C_{G}(P)$. Let $B_{\gamma}$ be a source algebra of $B$. Then the following assertions are equivalent:

(i) The ring extension $\mathrm{FP} \rightarrow \overline{B_{\gamma}}$ defined by the structural map $P \rightarrow \overline{B_{\gamma}} \times$ has depth two.

(ii) $B_{\gamma}$ is isomorphic to a twisted group algebra $R_{\sharp}[P \rtimes E]$ of the semidirect product $P \rtimes E$, as an interior $P$-algebra.

Proof. (i) $\Rightarrow$ (ii): Suppose that the ring extension $F P \rightarrow \overline{B_{\gamma}}$ has depth two, and write $A:=\overline{B_{\gamma}}=i F G i$, where $i$ is a primitive idempotent in $(F G)^{P}$. Then there exists a positive integer $n$ such that

$$
\operatorname{Res}_{F P}^{A} \operatorname{Ind}_{F P}^{A} \operatorname{Res}_{F P}^{A}(i M) \mid \operatorname{Res}_{F P}^{A}(i M)^{n}
$$

for every $B$-module $M$. Taking for $M$ the trivial $F G$-module $F$, we obtain

$$
A \otimes_{F P} i F \mid(i F)^{n}
$$

in ${ }_{F P}$ Mod. Thus, $P$ acts trivially on $A \otimes_{F P} i F$. On the other hand, $A$ is a direct sum of $(F P, F P)$-bimodules of the form $F[P g P]$, for suitable $g \in G$. It is easy to see that $F[P g P] \otimes_{F P} i F \cong \operatorname{Ind}_{P \cap g g^{-1}}^{P}(F)$ in ${ }_{F P}$ Mod. And if $P$ acts trivially on $\operatorname{Ind}_{P \cap g g^{-1}}^{P}(F)$, then $g \in N_{G}(P)$. Thus $A$ is in fact a direct sum of $(F P, F P)$ bimodules of the form $F[P g P]$, for suitable $g \in N_{G}(P)$. Hence [Thévenaz 1995, Theorem 44.3], a result by Puig, implies that $\operatorname{rk}_{R} B_{\gamma}=\operatorname{dim}_{F} \overline{B_{\gamma}}=|P| \cdot|E|$. Thus [Thévenaz 1995, Theorem 45.11], another result by Puig, implies (ii). 
(ii) $\Rightarrow$ (i): Suppose that (ii) holds. Since $R_{\sharp}[P \rtimes E]$ is a strongly $E$-graded ring with 1-component $R_{\sharp} P \cong R P$, [Boltje and Külshammer 2010, Proposition 1.5] shows that the ring extension $R P \rightarrow R_{\sharp}[P \rtimes E]$ has depth two. Tensoring with $F$, we obtain (i).

Remark 2.5. The implication (ii) $\Rightarrow$ (i) is valid for arbitrary blocks $B$ of $R G$. Also, if (ii) holds, one can show that every simple $\bar{B}$-module $M$ has trivial source by noting that $P$ acts trivially on $i M$.

\section{References}

[Benson 1998] D. J. Benson, Representations and cohomology, I: Basic representation theory of finite groups and associative algebras, 2nd ed., Cambridge Studies in Advanced Mathematics 30, Cambridge University Press, 1998. MR 99f:20001a Zbl 0908.20001

[Boltje and Glesser 2007] R. Boltje and A. Glesser, "On p-monomial modules over local domains", J. Group Theory 10:2 (2007), 173-183. MR 2007m:20013 Zbl 1179.20006

[Boltje and Külshammer 2010] R. Boltje and B. Külshammer, "On the depth 2 condition for group algebra and Hopf algebra extensions”, J. Algebra 323:6 (2010), 1783-1796. MR 2011a:16043 Zbl 1200.16035

[Curtis and Reiner 1981] C. W. Curtis and I. Reiner, Methods of representation theory, I, Wiley, New York, 1981. MR 82i:20001 Zbl 0469.20001

[Hertweck 2001] M. Hertweck, "A counterexample to the isomorphism problem for integral group rings", Ann. of Math. (2) 154:1 (2001), 115-138. MR 2002e:20010 Zbl 0990.20002

[Hirata 1969] K. Hirata, "Separable extensions and centralizers of rings", Nagoya Math. J. 35 (1969), 31-45. MR 39 \#5636 Zbl 0179.33503

[Kadison 1999] L. Kadison, New examples of Frobenius extensions, University Lecture Series 14, American Mathematical Society, Providence, RI, 1999. MR 2001j:16024 Zbl 0929.16036

[Kadison 2008] L. Kadison, "Finite depth and Jacobson-Bourbaki correspondence", J. Pure Appl. Algebra 212:7 (2008), 1822-1839. MR 2009f:16070 Zbl 1145.16021

[Kadison and Szlachányi 2003] L. Kadison and K. Szlachányi, "Bialgebroid actions on depth two extensions and duality”, Adv. Math. 179:1 (2003), 75-121. MR 2004i:16055 Zbl 1049.16022

[Külshammer 1991] B. Külshammer, Lectures on block theory, London Mathematical Society Lecture Note Series 161, Cambridge University Press, 1991. MR 92h:20020 Zbl 0726.20006

[Nagao and Tsushima 1989] H. Nagao and Y. Tsushima, Representations of finite groups, Academic Press, Boston, 1989. MR 90h:20008 Zbl 0673.20002

[Puig 1988] L. Puig, "Nilpotent blocks and their source algebras", Invent. Math. 93:1 (1988), 77116. MR 89e:20023 Zbl 0646.20010

[Thévenaz 1995] J. Thévenaz, G-algebras and modular representation theory, Oxford University Press, New York, 1995. MR 96j:20017 Zbl 0837.20015

Communicated by Dave Benson

Received 2010-01-15 Revised 2010-04-22 Accepted 2010-06-06

boltje@ucsc.edu

kuelshammer@uni-jena.de
Department of Mathematics, University of California, Santa Cruz, CA 95064, United States

Mathematical Institute, Friedrich Schiller University, 07737 Jena, Germany 


\section{Algebra \& Number Theory}

www.jant.org

\section{EDITORS}

MANAGING EDITOR

Bjorn Poonen

Massachusetts Institute of Technology

Cambridge, USA

\author{
EDITORIAL BOARD CHAIR \\ David Eisenbud \\ University of California \\ Berkeley, USA
}

BOARD OF EDITORS

Georgia Benkart

Dave Benson

Richard E. Borcherds

John H. Coates

J-L. Colliot-Thélène

Brian D. Conrad

Hélène Esnault

Hubert Flenner

Edward Frenkel

Andrew Granville

Joseph Gubeladze

Ehud Hrushovski

Craig Huneke

Mikhail Kapranov

Yujiro Kawamata

János Kollár

Yuri Manin

Barry Mazur

Susan Montgomery
University of Wisconsin, Madison, USA

University of Aberdeen, Scotland

University of California, Berkeley, USA

University of Cambridge, UK

CNRS, Université Paris-Sud, France

University of Michigan, USA

Universität Duisburg-Essen, Germany

Ruhr-Universität, Germany

University of California, Berkeley, USA

Université de Montréal, Canada

San Francisco State University, USA

Hebrew University, Israel

University of Kansas, USA

Yale University, USA

University of Tokyo, Japan

Princeton University, USA

Northwestern University, USA

Harvard University, USA

University of Southern California, USA
Shigefumi Mori

Andrei Okounkov

Raman Parimala

Victor Reiner

Karl Rubin

Peter Sarnak

Michael Singer

Ronald Solomon

Vasudevan Srinivas

J. Toby Stafford

Bernd Sturmfels

Richard Taylor

Ravi Vakil

Michel van den Bergh

Marie-France Vignéras

Kei-Ichi Watanabe

Andrei Zelevinsky

Efim Zelmanov
RIMS, Kyoto University, Japan

Princeton University, USA

Emory University, USA

University of Minnesota, USA

University of California, Irvine, USA

Princeton University, USA

North Carolina State University, USA

Ohio State University, USA

Tata Inst. of Fund. Research, India

University of Michigan, USA

University of California, Berkeley, USA

Harvard University, USA

Stanford University, USA

Hasselt University, Belgium

Université Paris VII, France

Nihon University, Japan

Northeastern University, USA

University of California, San Diego, USA

\section{PRODUCTION}

contact@msp.org

Silvio Levy, Scientific Editor

Andrew Levy, Production Editor

See inside back cover or www.jant.org for submission instructions.

The subscription price for 2011 is US \$150/year for the electronic version, and \$210/year (+ \$35 shipping outside the US) for print and electronic. Subscriptions, requests for back issues from the last three years and changes of subscribers address should be sent to Mathematical Sciences Publishers, Department of Mathematics, University of California, Berkeley, CA 94720-3840, USA.

Algebra \& Number Theory (ISSN 1937-0652) at Mathematical Sciences Publishers, Department of Mathematics, University of California, Berkeley, CA 94720-3840 is published continuously online. Periodical rate postage paid at Berkeley, CA 94704, and additional mailing offices.

ANT peer review and production are managed by EditFLOw ${ }^{\mathrm{TM}}$ from Mathematical Sciences Publishers.

PUBLISHED BY

mathematical sciences publishers

http://msp.org/

A NON-PROFIT CORPORATION

Typeset in LATEX

Copyright $\odot 2011$ by Mathematical Sciences Publishers 


\section{Algebra \& Number Theory}

\section{Volume $5 \quad$ No. $1 \quad 2011$}

Formules pour l'invariant de Rost

PhILIPPE GILle and ANNE QUÉGUINER-MATHIEU

Modular abelian varieties of odd modular degree

SOROOSH YAZDANI

Group algebra extensions of depth one

ROBERT BOLTJE and BURKHARD KÜLSHAMMER

Set-theoretic defining equations of the variety of principal minors of symmetric matrices

LUKE OEDING

Frobenius difference equations and algebraic independence of zeta values in positive equal characteristic

Chieh-Yu Chang, Matthew A. Papanikolas and Jing Yu 